\title{
Quantitative analysis of airway obstruction in lymphangioleiomyomatosis
}

\author{
Stijn E. Verleden ${ }^{1,9}$, Arno Vanstapel $^{1,9}$, Laurens De Sadeleer (10) \\ Birgit Weynand $\mathbb{1}^{2}$, Matthieu Boone ${ }^{3}$, Erik Verbeken ${ }^{2}$, Davide Piloni ${ }^{4}$, \\ Dirk Van Raemdonck ${ }^{5}$, Maximilian Ackermann $\mathbb{1}^{6,8}$, Danny D. Jonigk ${ }^{7}$, \\ Johny Verschakelen ${ }^{2}$ and Wim A. Wuyts (1) ${ }^{1}$
}

\begin{abstract}
Affiliations: ${ }^{1}$ Respiratory Diseases, Dept of Chronic Diseases, Metabolism and Aging, KU Leuven, Leuven, Belgium. ${ }^{2}$ Dept of Imaging, KU Leuven, Leuven, Belgium. ${ }^{3}$ Dept of Physics and Astronomy, Radiation PhysicsCentre for X-ray Tomography, Ghent University, Ghent, Belgium. ${ }^{4}$ The Respiratory Disease Unit, Fondazione IRCCS Policlinico San Matteo, University of Pavia, Pavia, Italy. ${ }^{5}$ Thoracic Surgery, Dept of Chronic Diseases, Metabolism and Aging, KU Leuven, Leuven, Belgium. 'Institute of Functional and Clinical Anatomy, University Medical Center of the Johannes Gutenberg-University Mainz, Mainz, Germany. ${ }^{7}$ Institute of Pathology, Hannover Medical School, Hannover, Germany. ${ }^{8}$ Institute of Pathology and Molecular Pathology, Helios University Hospital Wuppertal, Witten-Herdecke University, Wuppertal, Germany. ${ }^{9}$ Both authors contributed equally.
\end{abstract}

Correspondence: Stijn E. Verleden, KU Leuven, Lung Transplantation Unit, 49 Herestraat, B-3000 Leuven, Belgium. E-mail: stijn.verleden@med.kuleuven.be

@ERSpublications

This study demonstrates a 4-fold reduction in the number of airways and terminal bronchioles in endstage LAM lungs using a combination of CT, microCT and histopathology, compared to a matched control group http://bit.ly/2tBTiJy

Cite this article as: Verleden SE, Vanstapel A, De Sadeleer L, et al. Quantitative analysis of airway obstruction in lymphangioleiomyomatosis. Eur Respir J 2020; 56: 1901965 [https://doi.org/10.1183/ 13993003.01965-2019].

ABSTRACT Lymphangioleiomyomatosis (LAM) is a rare, cystic lung disease with progressive pulmonary function loss caused by progressively proliferating LAM cells. The degree of airway obstruction has not been well investigated within the pathogenesis of LAM.

Using a combination of ex vivo computed tomography (CT), microCT and histology, the site and nature of airway obstruction in LAM explant lungs was compared with matched control lungs ( $\mathrm{n}=5$ each). The total number of airways per generation, total airway counts, terminal bronchioles number and surface density were compared in LAM versus control.

Ex vivo CT analysis demonstrated a reduced number of airways from generation 7 on $(\mathrm{p}<0.0001)$ in LAM compared with control, whereas whole-lung microCT analysis confirmed the three- to four-fold reduction in the number of airways. Specimen microCT analysis further demonstrated a four-fold decrease in the number of terminal bronchioles $(p=0.0079)$ and a decreased surface density $(p=0.0079)$. Serial microCT and histology images directly showed the loss of functional airways by collapse of airways on the cysts and filling of the airway by exudate.

LAM lungs show a three- to four-fold decrease in the number of (small) airways, caused by cystic destruction which is the likely culprit for the progressive loss of pulmonary function.

This article has an editorial commentary: https://doi.org/10.1183/13993003.02162-2020

Received: 7 Oct 2019 | Accepted after revision: 3 Feb 2020

Copyright CERS 2020. This version is distributed under the terms of the Creative Commons Attribution NonCommercial Licence 4.0. 


\section{Introduction}

Lymphangioleiomyomatosis (LAM) is a rare disease targeting the lung, mostly occurring in women, where the lung is slowly destroyed by cystic destruction, ultimately leading to respiratory failure over a period from 10 to 20 years. Diagnosis is often complicated given the rarity of the disease leading to confusion with more common diseases such as COPD or asthma. The decline in pulmonary function is highly variable (3-15\% per year). Symptoms include recurrent pneumothorax, pleural effusions and abdominal tumours [1].

Invading LAM cells, which are alpha smooth muscle like, are believed to be the main culprit for the disease. These cells infiltrate the lung and stimulate the degradation of the matrix leading to cell death and eventual cyst formation, although a role for disorganised lymphangiogenesis has also been put forward as one of the key mechanisms. The proliferation of LAM cells along the lymphatic puts them close to the airways and blood vessels. Obstruction of blood vessels can result in areas of haemorrhage, while obstruction of lymphatics can lead to chylothorax. In addition, the constrictive effect of bundles of LAM cells on airways can result in airflow obstruction, leading to air trapping and ultimately cystic changes in the pulmonary parenchyma [2], evidenced by the association between the severity of cystic changes and air trapping [3].

Standard pulmonary function tests mostly show a mixed obstructive and restrictive pulmonary function defect. The obstructive pulmonary function defect has been attributed to airway narrowing and obstruction based on maximal flow-static recoil curves [4]. Investigation of three LAM autopsy lungs revealed that small airways were narrowed and collapsed because of the surrounding cysts cause a loss of support of the airways. Intriguingly, this was not accompanied by an increased distribution of the smooth muscles in these airways [5]. A more recent histopathological study focussing on the airways in LAM found a bronchial involvement by LAM cells and lymphatics in all 30 investigated patients, which was accompanied by chronic inflammation, goblet cell hyperplasia, squamous cell metaplasia of the epithelium and thickening of the basal lamina in the majority of the investigated cases [6]. However, the degree of airway inflammation did not correlate with the severity of airflow obstruction [7].

Although there is a lot of evidence that the airways are the cause of airway obstruction, probably secondary to cyst formation, quantitative evidence of the degree of airway involvement and the exact nature of the airway obstruction has not been well investigated in LAM. We have previously investigated the site and nature of airway obstruction in obstructive diseases such as COPD, bronchiolitis obliterans syndrome post-lung transplantation and cystic fibrosis [8-10]. Therefore, the aim of the current study was to provide quantitative information regarding the pathogenesis of airway obstruction in LAM by a thorough morphometric comparison to matched control lungs.

\section{Material and methods \\ Patient selection}

Explant lungs were collected from patients undergoing lung transplantation for end-stage LAM at UZ Gasthuisberg, Leuven, Belgium. Unused donor lungs were matched according to age and sex and included as controls. All patients provided written informed consent and this study was approved by the hospitals ethical committee (S52174). Donor lungs were collected following Belgian legislation where declined donor lungs can be offered for research (S59648, S61653).

\section{Lung processing for ex vivo computed tomography and whole lung microCT}

Lungs were processed as described previously [7]. In brief, lung were air-inflated at $30 \mathrm{~cm} \mathrm{H}_{2} \mathrm{O}$ and after deflation to $10 \mathrm{~cm} \mathrm{H}_{2} \mathrm{O}$, lungs were fixed in the fumes of liquid nitrogen at constant airway pressure and preserved at $-80^{\circ} \mathrm{C}$ and scanned with ex vivo computed tomography (CT) in frozen conditions (Siemens Somatom, $1.0 \mathrm{~mm}$ slice thickness, B60F window). This CT was used to manually count the number of airways per generation [8, 11]. Additionally, lung volume, lung density and lung weight were determined using Horos (open source code software (FOSS) program at Horosproject.org and sponsored by Nimble Co LLC d/b/a Purview in Annapolis, MD, USA). To improve the spatial resolution, from two explants (one LAM and one control), the contralateral (right) explant lung was separated in the upper/middle and lower lobe. Both lobes were separately air-inflated and air-dried for 1 week using constant air pressure. The dried specimens were then scanned using the in-house developed microCT system HECTOR [12] operated at $120 \mu \mathrm{m}$ voxel size, tube voltage $80 \mathrm{kV}, 208 \mu \mathrm{A}$ and reconstructed using Octopus Reconstruction (XRE, Ghent, Belgium). These images were loaded into ITK-SNAP for semi-automated segmentation of the airways [13]. NEURONstudio was used to assess the number of airways per generation and the total airway count [14].

\section{MicroCT and histology}

After CT scans, lungs were sliced using a bandsaw from apex to base and were systematically sampled using a core bore or power drill resulting in specimen cores $(2 \mathrm{~cm}$ height, $1.4 \mathrm{~cm}$ diameter). We randomly selected four cores (two apical, two basal) per lung, which were scanned in frozen condition $\left(-30^{\circ} \mathrm{C}\right)$ using microCT 
(Skyscan 1172, Bruker, Kontich, Belgium) at a voxel size of $10 \mu \mathrm{m}$. This subsample microCT scan was further used to manually count the number of terminal bronchioles (defined anatomically as the last conducting airway) and measure the airway wall thickness on three random terminal bronchioles per lung sample (if fewer than three terminal bronchioles were present, all remaining terminal bronchioles were assessed). To measure surface density, tissue volume and tissue percentage, CTan was used (Bruker, Kontich, Belgium).

Following frozen microCT, a selected sample was fixed in 6\% paraformaldehyde, dehydrated using ethanol, embedded in paraffin and sectioned at $5 \mu \mathrm{m}$ slice thickness and stained with haematoxylin and eosin (H\&E), elastica von Giesson and Human Melanoma Black (HMB) 45 according to conventional protocols.

\section{Scanning electron microscopy and vascular corrosion casting}

Briefly, the afferent vessels were cannulated with an olive-tipped cannula. The vasculature was flushed with saline (at body temperature) followed by glutaraldehyde fixation solution (2.5\%, pH 7.4; Sigma Aldrich, Munich, Germany). Fixation was followed by injection of prepolymerised PU4ii resin (VasQtec, Zurich, Switzerland) mixed with a hardener ( $40 \%$ solvent) and blue dye as casting medium. After curing of the resin, the lung tissue was macerated in $10 \% \mathrm{KOH}$ (Fluka, Neu-Ulm, Germany) at $40^{\circ} \mathrm{C}$ for $2-3$ days. Specimens were then rinsed with water and frozen in distilled water. The casts were freeze-dried and sputtered with gold in an argon atmosphere and examined using a Philips ESEM XL-30 scanning electron microscope at $15 \mathrm{keV}$ and $21 \mu \mathrm{A}$ (Philips, Eindhoven, the Netherlands).

\section{Statistical analysis}

Results are expressed as median and interquartile range (IQR) or mean \pm SD. The number of terminal bronchioles, the surface density and percentage of tissue were averaged over the entire lung. Differences between the LAM and control group were compared using a Wilcoxon signed rank test. The number of airways per generation was compared using two-way ANOVA with Tukey's post hoc test (Graphpad Prism 4.0; Graphpad, CA, USA). A p-value $<0.05$ was considered significant.

\begin{tabular}{|c|c|c|c|}
\hline & control & LAM & p-value \\
\hline Subjects $\mathrm{n}$ & 5 & 5 & \\
\hline Age years & $51 \pm 8$ & $46 \pm 12$ & 0.42 \\
\hline Sex female $n(\%)$ & $5(100)$ & $5(100)$ & 1.00 \\
\hline Height $\mathrm{cm}$ & $166 \pm 4$ & $163 \pm 4$ & 0.35 \\
\hline Weight kg & $68 \pm 14$ & $66 \pm 8$ & 1.00 \\
\hline Smoking history (non-/ex smoker) n & $4 / 1$ & $4 / 1$ & 1.00 \\
\hline Time between diagnosis and LTx years & NA & $8.5 \pm 5.0$ & \\
\hline \multicolumn{4}{|l|}{ Pulmonary function } \\
\hline $\mathrm{FEV}_{1} \mathrm{~L}$ & NA & $1.0 \pm 0.43$ & \\
\hline $\mathrm{FEV}_{1} \%$ & NA & $37 \pm 14$ & \\
\hline FVC L & NA & $2.6 \pm 0.7$ & \\
\hline FVC \% & NA & $83 \pm 17$ & \\
\hline $\mathrm{FEV}_{1} / \mathrm{FVC}$ & NA & $0.38 \pm 0.11$ & \\
\hline TLC L & NA & $6.0 \pm 1.0$ & \\
\hline TLC \% & NA & $120 \pm 19$ & \\
\hline$D_{\mathrm{LCO}} \mathrm{L}$ & NA & $2.4 \pm 0.6$ & \\
\hline$D_{\mathrm{Lco}} \%$ & NA & $28 \pm 7$ & \\
\hline \multicolumn{4}{|l|}{ CT measurement } \\
\hline Lung volume $L$ & $2.47 \pm 0.50$ & $3.13 \pm 0.44$ & 0.21 \\
\hline Lung density $\mathrm{g} \cdot \mathrm{L}^{-1}$ & $128 \pm 21$ & $189 \pm 57$ & 0.095 \\
\hline Lung weight g & $318 \pm 38$ & $600 \pm 235$ & 0.095 \\
\hline \multicolumn{4}{|l|}{ MicroCT measurement } \\
\hline $\mathrm{TB} / \mathrm{mL}$ & $5.06 \pm 1.89$ & $1.06 \pm 0.16$ & 0.0079 \\
\hline TB/lung & $12510 \pm 4268$ & $3296 \pm 363$ & 0.0079 \\
\hline Diameter TB $\mu \mathrm{m}$ & $386 \pm 35$ & $368 \pm 68$ & 0.84 \\
\hline Surface density $1 / \mu \mathrm{m}$ & $0.11 \pm 0.02$ & $0.03 \pm 0.01$ & 0.0079 \\
\hline Tissue percentage $\%$ & $37.8 \pm 2.6$ & $23.4 \pm 4.7$ & 0.0079 \\
\hline Wall thickness $\mu \mathrm{m}$ & $71.8 \pm 5.9$ & $154.1 \pm 45.9$ & 0.0079 \\
\hline
\end{tabular}

Data are presented as mean $\pm \mathrm{SD}$, unless otherwise stated. $\mathrm{LAM}$ : lymphangioleiomyomatosis; $\mathrm{FEV}_{1}$ : forced expiratory volume in $1 \mathrm{~s}$; FVC: forced vital capacity; TLC: Total lung capacity; $D_{\text {Lco }}$ : diffusing capacity of the lung for carbon monoxide; CT: computed tomography; TB: terminal bronchiole. Bold text indicates statistical significance. 


\section{Results}

\section{Patient characteristics}

We included five LAM lungs in this analysis; the LAM patients' median age was $46 \pm 12$ years, they were exclusively female with only one former smoker and were well matched with our control group $(n=5)$ (table 1). Mean time from disease diagnosis to transplant was 8.5 years (range 3.3-15.2 years). Last pulmonary function test before transplantation was compatible with end-stage pulmonary disease with especially a very low diffusion capacity. A comparative view of a representative LAM and control lung are shown in figure 1, where especially the cyst formation in the LAM lungs is evident from gross inspection and CT.

\section{Ex vivo CT and whole lung microCT analysis}

First, the number of airways per generation was quantified manually based on the frozen lung ex vivo CT (resolution $0.7-1 \mathrm{~mm}$ ). This demonstrated a significant reduction in the number of airways compared to the control group $(p<0.0001)$, with especially decreases in the number of airways from generation 7 $(\mathrm{p}=0.070), 8(\mathrm{p}=0.035), 9(\mathrm{p}=0.015)$ and $10(\mathrm{p}=0.065)$ compared with the control group (figure $2 \mathrm{a})$. The absolute number of airways was lower in LAM lungs (182 \pm 49$)$ compared with control lungs $(347 \pm 202)$ $(\mathrm{p}=0.016)$. The ex vivo CT further demonstrated an increased lung volume, lung density and lung mass, although none reached statistical significance (table 1). Of note is also the small standard deviation in these variables in LAM lungs compared with control, for which there was much more heterogeneity in the number of airways per generation and the total airway number, indicating that the disease was homogeneous across the disease specimens.

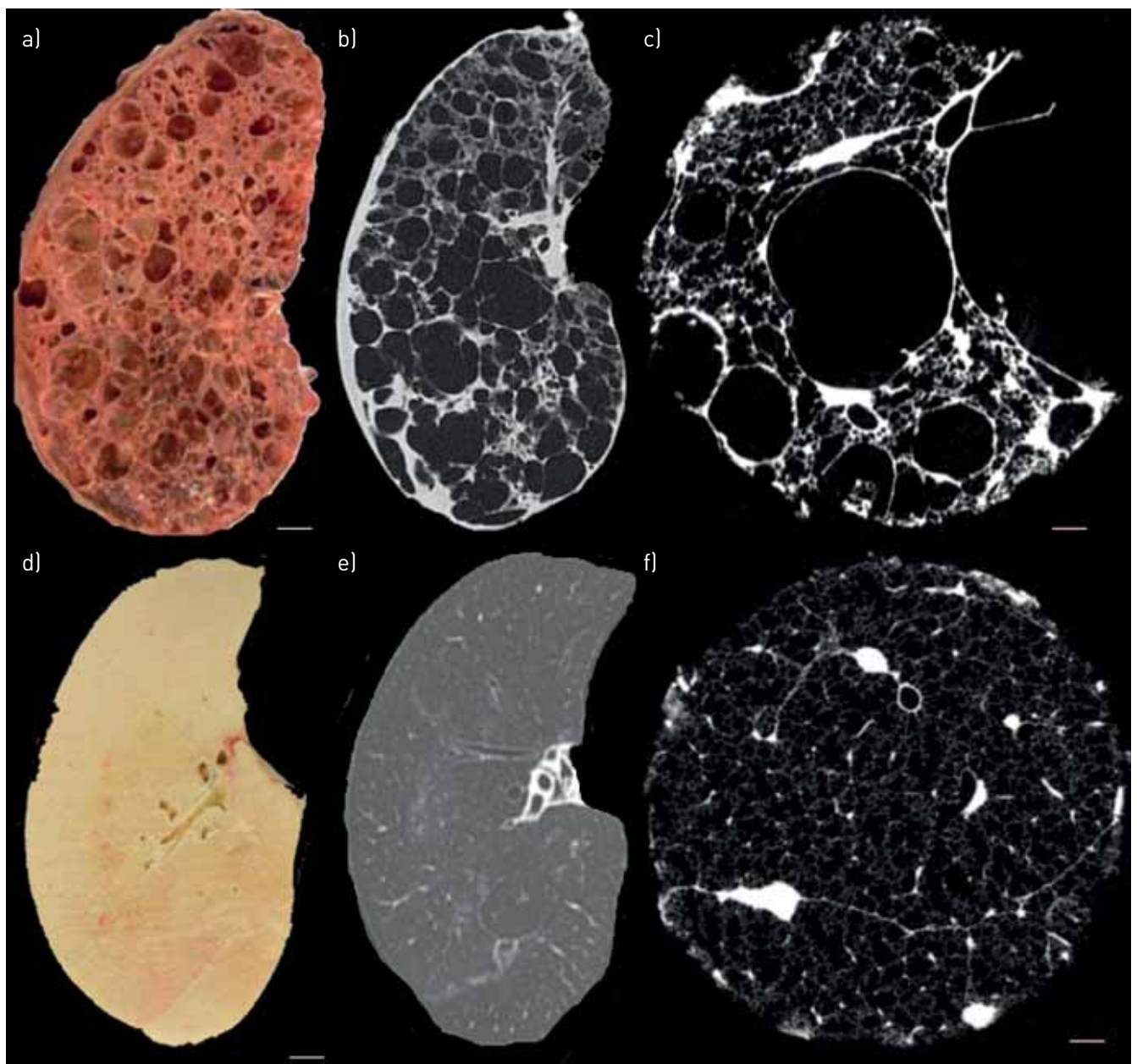

FIGURE 1 Comparison of lymphangioleiomyomatosis (LAM) and control lung tissue. a) Gross slice image of a LAM lung. b) Matched ex vivo computed tomography (CT) image in LAM. c) Representative subsample microCT image demonstrating evidence of cysts adjacent with limited normal surrounding tissue. d) Gross image of control lung. e) Ex vivo CT image of control lung at the same location. f) MicroCT image in control lung demonstrated normal lung tissue. al and d) Scale bars=1 cm; c) and f) Scale bars: $1 \mathrm{~mm}$. 

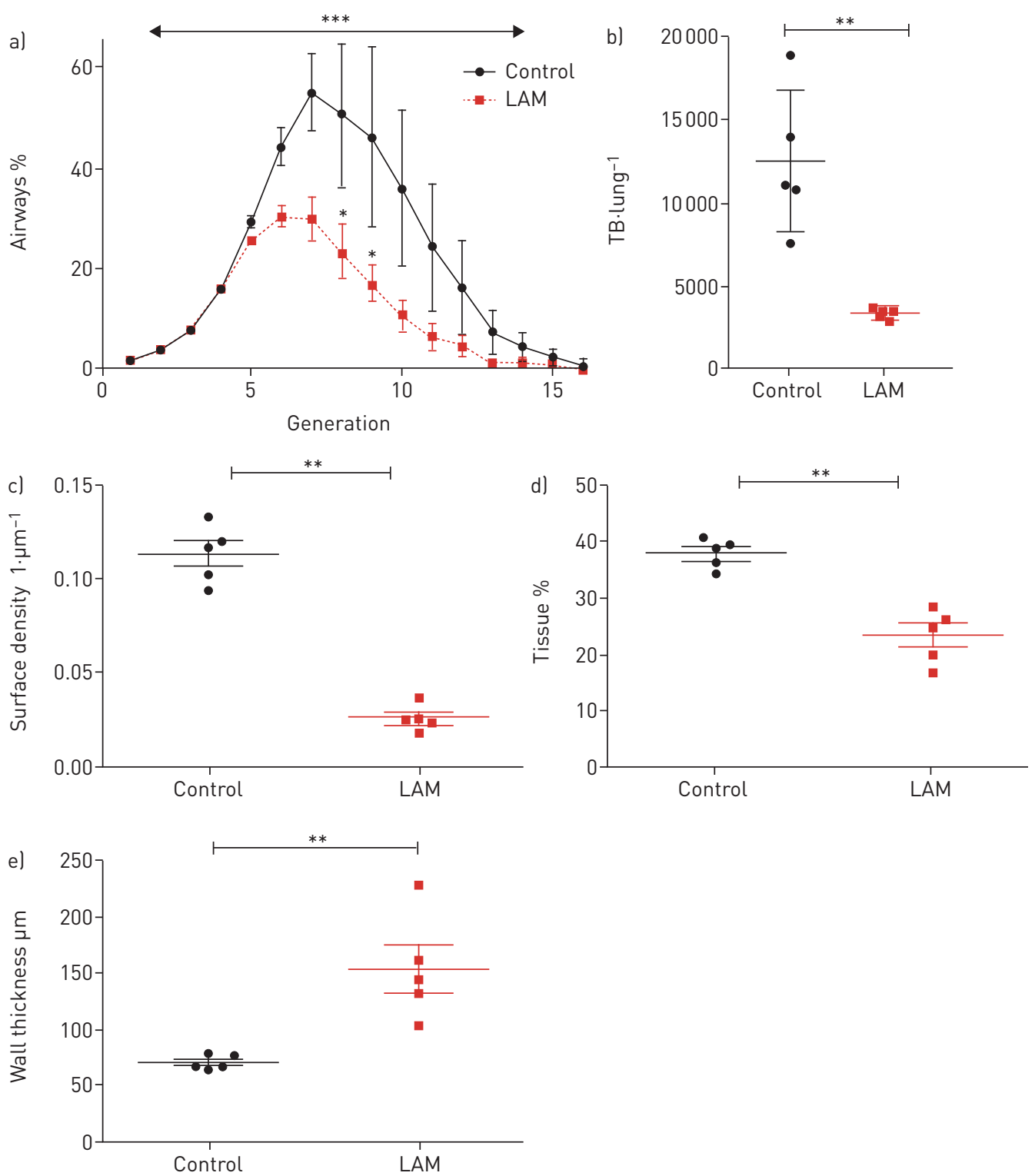

FIGURE 2 Conventional computed tomography (CT)-based airway measures. a) The number of airways per generation is lower in lymphangioleiomyomatosis (LAM) compared to control from generation 7 till 10. b) The number of terminal bronchioles (TB) was decreased in LAM compared with control. c) Surface density is lower in LAM compared with control. d) Tissue percentage is decreased in LAM compared with control. e) Wall thickness of the terminal bronchioles in increased in LAM compared with control. **: $p<0.01$.

Given the limited resolution of the ex vivo CT protocol $(0.7-1 \mathrm{~mm})$, state of the art whole-lung microCT was performed $(120 \mu \mathrm{m}$ resolution) to increase the visualisation of especially the smaller airways. The airway casts provide a visual and quantitative impression of the loss of airways in LAM (figure 3a and b), in which 2074 airways were detected in the control lung which was reduced three-fold in LAM lungs to 695 airways. This loss of airways was specifically apparent from generation 7 and was consistent throughout all subsequent airway generations (figure 3c), confirming our previous CT-based approach. Stratifying the number of airways according to their diameter demonstrated that especially small-sized airways are lost (figure 3d). However, considering the percentage of airways with a certain diameter (proportionally to the total number of airways), it is evident that there is a general loss of airways irrespective of their diameter (figure $3 \mathrm{e}$ ).

\section{Subsample microCT analysis}

Next, microCT was used on extracted specimens to quantify the number of terminal bronchioles, the last conduction airway before the alveoli (resolution $10 \mu \mathrm{m}$ ). MicroCT analysis demonstrated a five-fold 


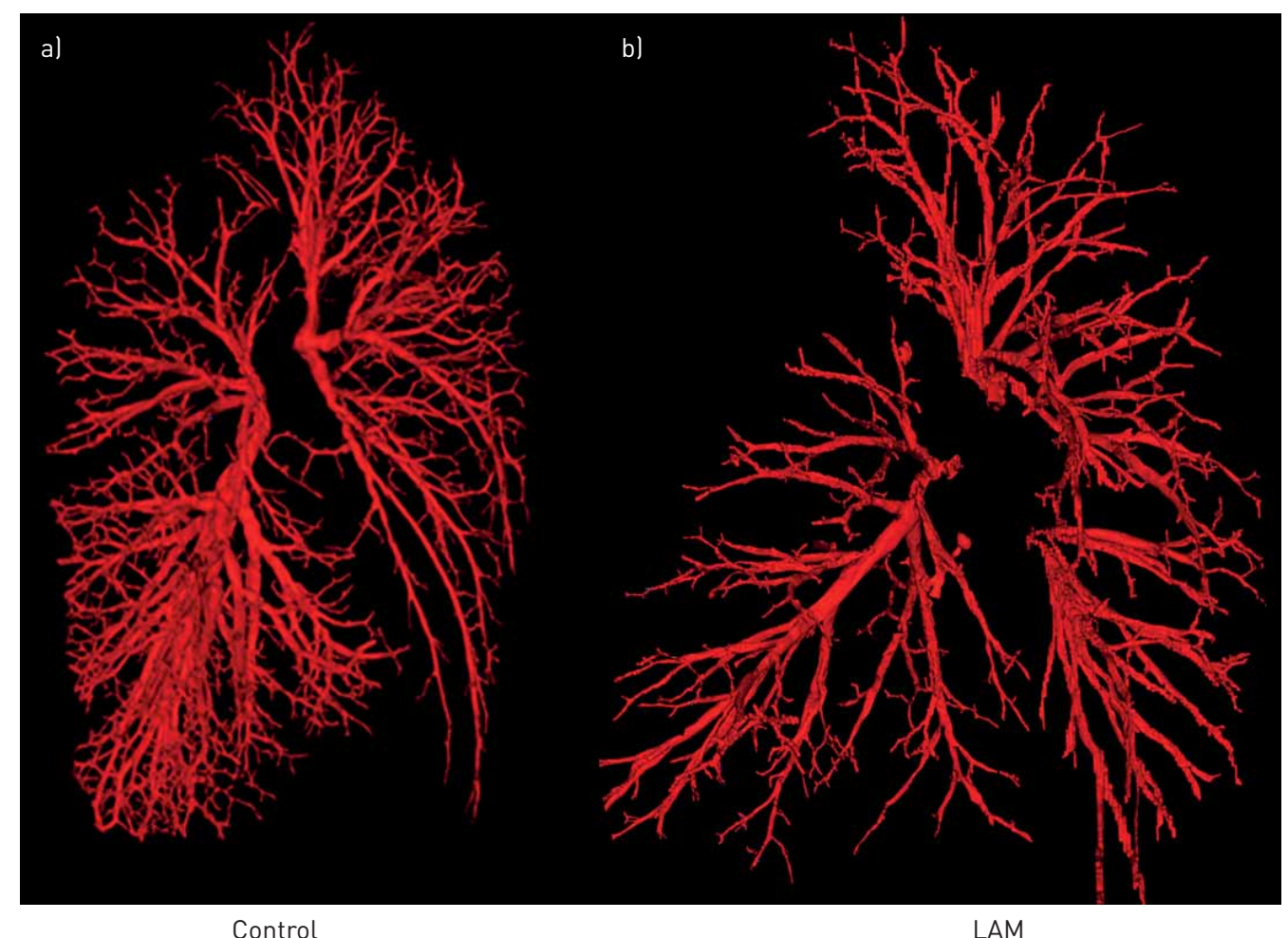

Control

LAM

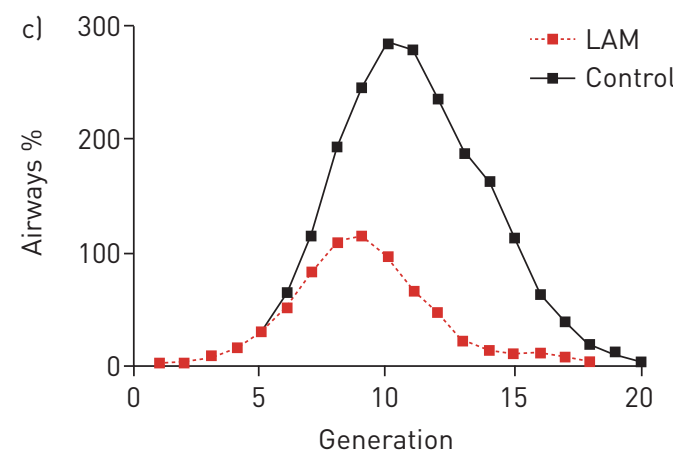

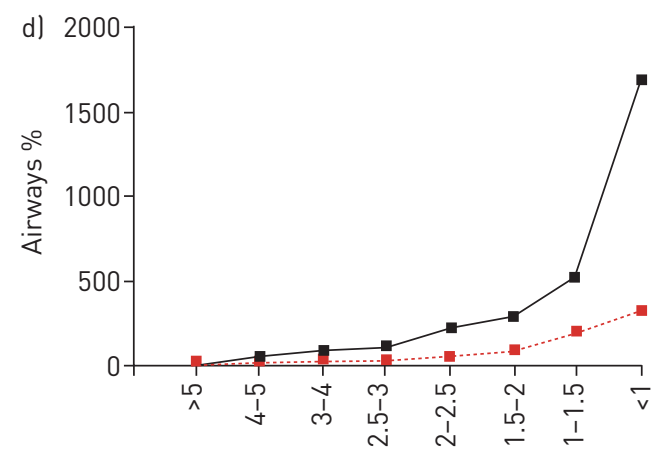

Airway diameter $\mathrm{mm}$

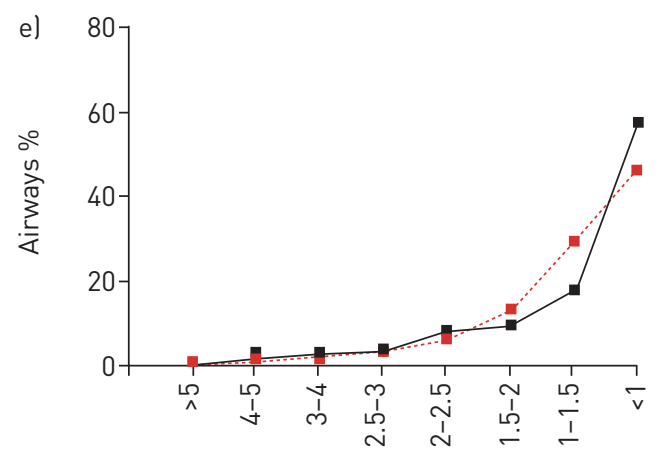

Airway diameter $\mathrm{mm}$

FIGURE 3 Whole lung micro-computed tomography (microCT) based airway measures. a) Complete airway segmentation of a right control lung. b) Airway segmentation of a right lymphangioleiomyomatosis (LAM) lung. c) The number of airways per generation based on whole lung microCT is lower in LAM compared with control. d) The number of airways per airway diameter. e) The proportion of airways relative to the total airway number per generation.

decrease in the number of terminal bronchioles per $\mathrm{mL}$ of tissue $(\mathrm{p}=0.0079)$ and a four-fold decrease in the total number of terminal bronchioles per lung $(\mathrm{p}=0.0079)$. The diameter of the remaining terminal bronchioles did not differ between the LAM and control group, while the airway wall of the terminal 

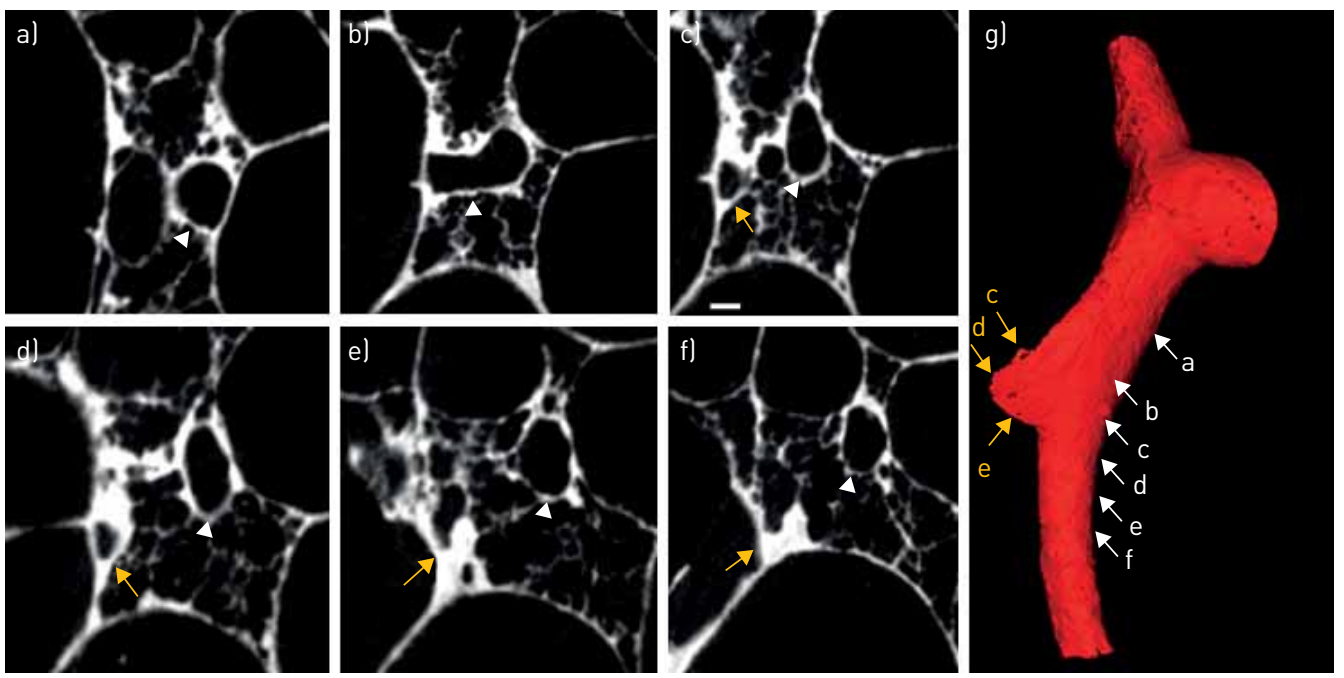

FIGURE 4 Serial subsample micro-computed tomography (microCT) images demonstrating collapse of airways on cystic structure. a) Mother branch. b) Bifurcation. c) Two separate airways. d) Airway lumen decrease in one airway (yellow), intact lumen in the other (white). e) Progressive luminal loss. f) Total luminal obliteration with an adjacent patent airway. g) 3D microCT illustration.

bronchioles was significantly thicker in LAM compared to control $(\mathrm{p}=0.0079)$. The surface density as a measure of the parenchymal integrity demonstrated a pronounced decrease in LAM compared to control $(\mathrm{p}=0.0079)$, consistent with an increased distance between the alveoli due to cyst formation and dilatation of the parenchyma. Moreover, the number of terminal bronchioles was directly related to the surface density ( $\mathrm{p}=0.0015, \mathrm{R}=0.66$ ), indicating that more preserved lung parenchyma (i.e. higher surface density) harbour more terminal bronchioles per $\mathrm{mL}$ of tissue.

Within the microCT scans, direct evidence of airway narrowing and loss was found. Figure 4 demonstrates serial microCT images demonstrating a mother branch (figure 4a) bifurcating in two daughter branches (figure $4 \mathrm{~b}$ and $\mathrm{c}$ ) with one branch remaining intact with a normal airway lumen diameter (white arrow), while the other airway (orange) collapses on to the adjacent parenchymal cyst where the lumen is completely lost and not restored in subsequent serial microCT images (figure $4 \mathrm{~d}-\mathrm{f}$ ). The $3 \mathrm{D}$ reconstruction clearly demonstrates a sudden stop of the airway integrity (figure $4 \mathrm{~g}$ ). Matching serial microCT images with H\&E, elastica Von Giesson staining and HMB45 staining confirmed the gradual complete compression of the bronchial lumen by cyst formation and furthermore demonstrated abundant elastin fibres and ample presence and infiltration of HMB45+ LAM cells (magenta stained cells) in the walls of the compressed airways (figure 5). Next to the loss of airways, due to collapse on the cysts, airways were also filled with exudate as shown in figure 6. The lumen of an intact airway (figure 6a), decreases progressively (figure $6 \mathrm{~b}$ and $\mathrm{c}$ ), until the lumen is not discernible anymore and is completely filled (figure $6 \mathrm{~d}$ ). The $3 \mathrm{D}$ reconstruction (figure 6e) confirms the progressive narrowing of the lumen and the subsequent stop of the airway after the completely filled airway segment. Although, cysts are present around this airway, the microCT (figure 6f) and histology (figure 6g) images demonstrate filling of the airway lumen by a mixture of oedema, fibrin and blood (figure $6 \mathrm{~h}$ ), an exudate which is also present in the adjacent alveolar parenchyma, together with haemosiderin-loaded macrophages (figure 4i), suggestive of chronic/repetitive bleeding.

To assess the specificity of the cystic destruction, we used a combination of scanning electron microscopy and microvascular casting to further investigate the structural morphology and vascularity of LAM demonstrating the typical cystic destruction (figure $7 \mathrm{a}-\mathrm{d}$ ) and furthermore a distinct aberrant vascularity with sinusoid-like vessel and missing hierarchy (figure 7e and f).

\section{Discussion}

This study investigated the site and nature of airway obstruction in LAM and demonstrated an almost four-fold reduction in the number of airways visible on CT and whole lung microCT. Subsample microCT furthermore confirmed a four-fold reduction in the number of terminal bronchioles where functional airways were lost due to collapse of the airway on the typical LAM cysts, but also due to progressive filling of the airways with exudate, which was further confirmed by histology.

This report confirms and substantially extends previous work that has been performed investigating the site of airway obstruction in LAM. SoBonya et al. [5] already proposed that the airspace lesions observed 

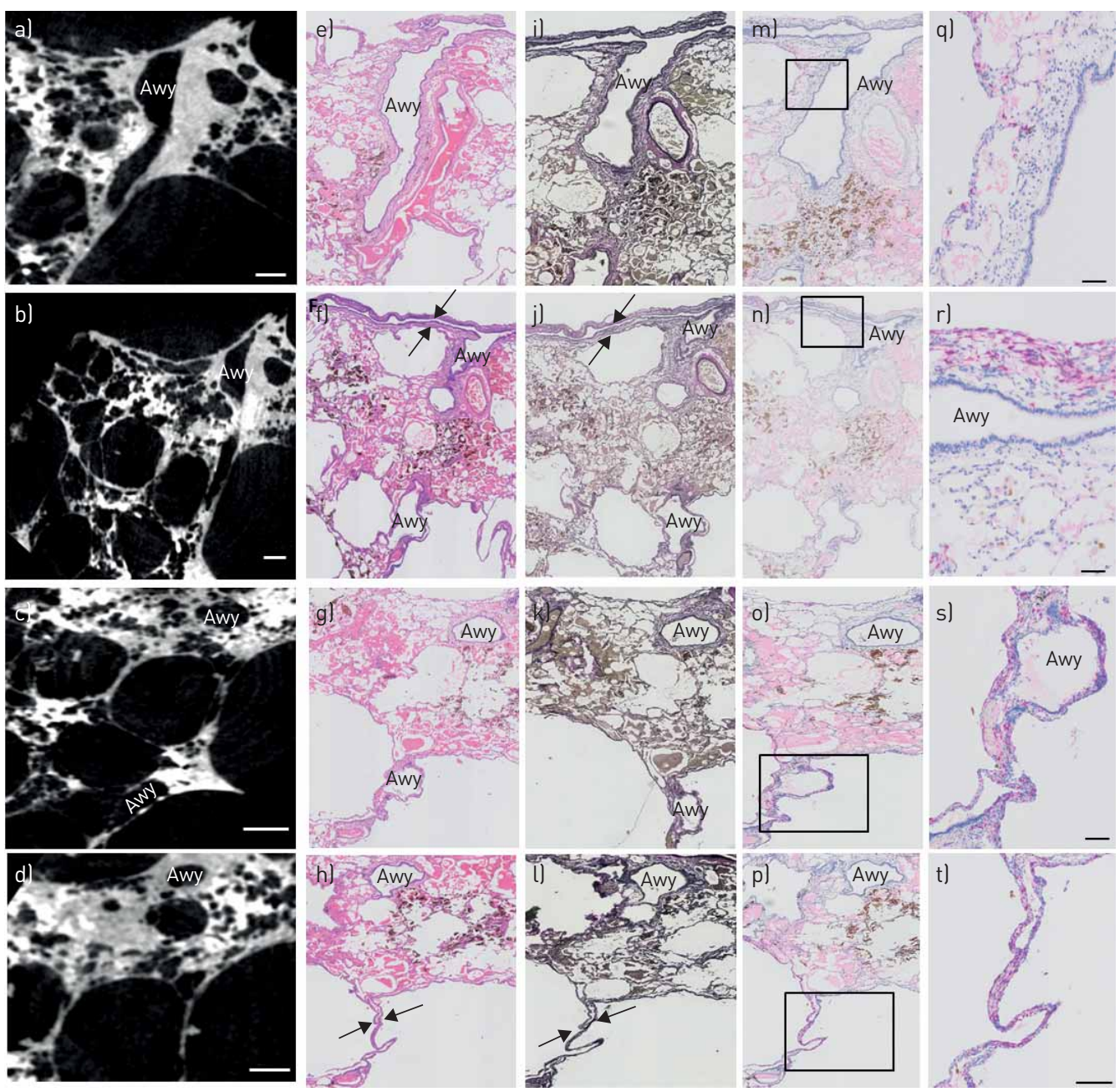

FIGURE 5 Comparison of serial micro-computed tomography (microCT) images (a-d) with matched haematoxylin-eosin (H\&E) (e-h), Elastica van Gieson (EvG) (i-l), and HMB45 (m-t) stained serial sections. MicroCT imaging initially displays an open bronchus (a), where a bronchus is compressed (b) and where additional serial sectioning demonstrates further compression of the lumen (c-d). The corresponding H\&E and EvG stains confirm the gradual complete compression of the bronchial lumen by cyst formation larrows in $f$ and $h$ ), as evidenced by preservation of the bronchial epithelium between these cysts. In addition, there are signs of severe haemorrhage, with abundant presence of red blood cells and haemosiderin-laden macrophages. The corresponding HMB45 images show a preserved airway wall in a patent bronchus $(\mathrm{m}$ and q), but abundant presence and infiltration of HMB45+ lymphangioleiomyomatosis (LAM) cells (magenta stained cells) in the walls of the compressed airways ( $r-t)$, potentially contributing to the airway obliteration with even complete loss of the airway lumen (t). Awy: airway. Scale bars: (a-d) $500 \mu \mathrm{m}$; (q-t) $100 \mu \mathrm{m}$.

in LAM are more important in the genesis of the airflow limitation rather than the airways themselves. We extend their initial observation as we observed a four-fold reduction in the (small) airways, mostly caused by collapse of the airway or filling of the airway by exudate. The abundant presence of HMB45-positive cells in the airway wall of the compressed airways suggests a potential contribution of these cells to the progressive compression of the lumen between the cysts. We speculate that the abundant exudate/chronic alveolar bleeding is due to the cystic destruction of the alveolar parenchyma with prominent secretion retention. This very likely is the explanation of the haemoptysis typically found in LAM patients.

The observed loss in airways is reminiscent of the degree of airway loss in COPD. McDonougH et al. [10] showed a 72 to $89 \%$ reduction in the number of terminal bronchioles in GOLD IV compared with a $75 \%$ reduction observed in this study for LAM patients. The underlying mechanism of terminal bronchiolar loss however seems to be different. In emphysema, there is also a reduction in terminal bronchiolar dimensions, while the remaining terminal bronchioles have normal dimensions in LAM. This is likely explained by the different pathophysiological mechanisms where the exposure to cigarette smoke causes disruption and injury to the airway epithelium leading to progressive narrowing and loss of the terminal bronchioles in emphysema; in LAM the airway loss is explained by progressive matrix degradation around 

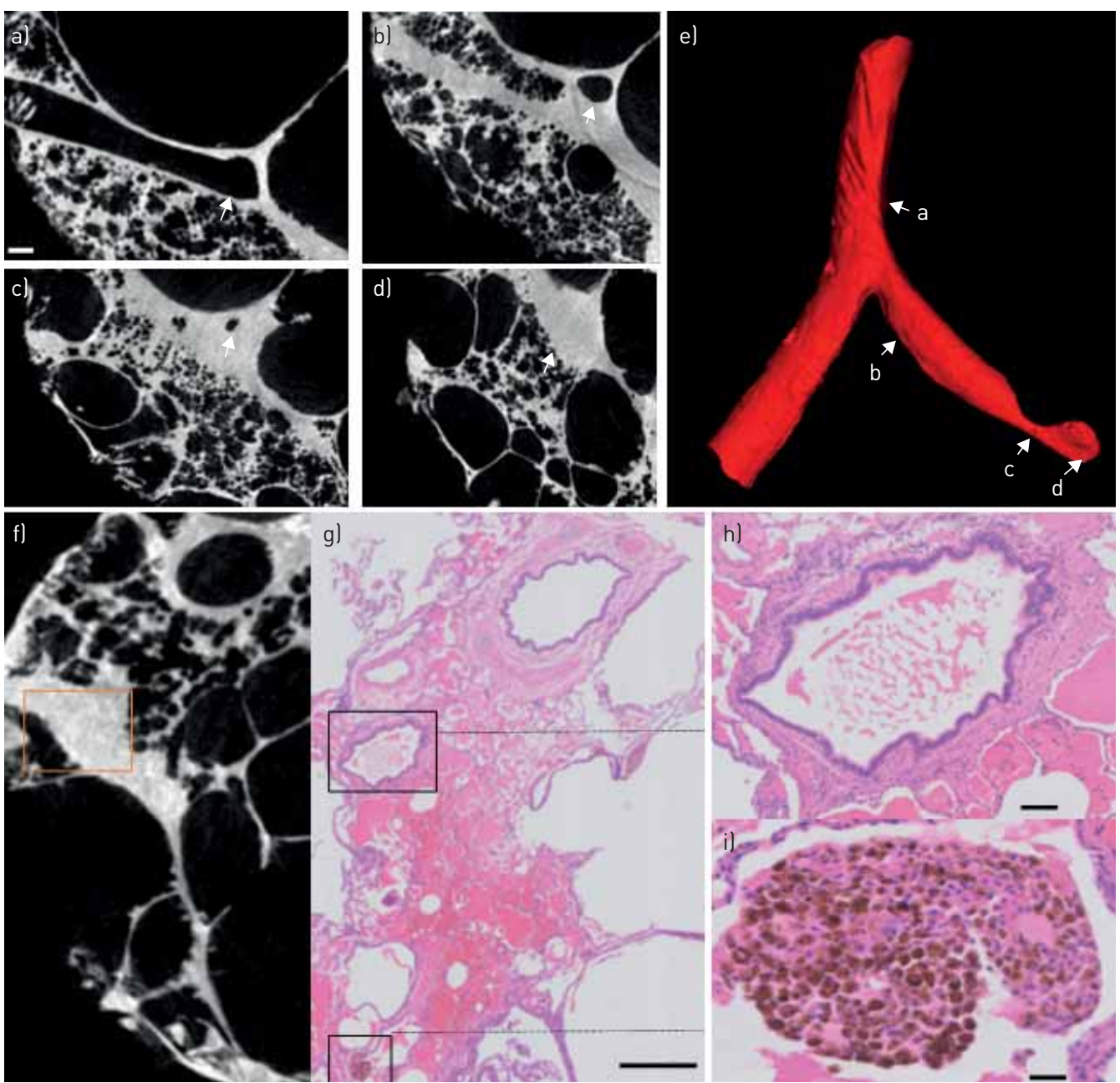

FIGURE 6 Serial subsample micro-computed tomography (microCT) images demonstrating airway loss due to fibrosis. a) Mother branch. b) Airway with peribronchiolar fibrosis. c) Luminal narrowing. d) Luminal loss in region of fibrosis. e) 3D reconstruction demonstrating airway obstruction. f) MicroCT image showing a patent and obstructed bronchiole (highlighted with the rectangle). g) Corresponding haematoxylin-eosin (H\&E) image showing filling of the airway with exudate. h) Higher magnification image showing filling of the airway. i) Evidence of haemosiderin-filled macrophages in close proximity of the airway. Scale bars: (g) $800 \mu \mathrm{m}$; (h) $100 \mu \mathrm{m}$; (i) $50 \mu \mathrm{m}$.

the airways, where the airway progressively loses its support and eventually collapses on the cystic structures. The question remains to be solved whether all airways were truly lost or some of the airways failed to grow.

There is probably an important role for airway inflammation as LAM patients with more advanced disease appear to have chronic inflammation [6], although it is very difficult to separate cause and consequence. Whether inflammation is a primary culprit or a second hit is yet unknown: indeed the impaired airway integrity due to the cystic deformation of the parenchyma can make it more prone to bronchial inflammation or excessive infiltration of LAM cells can cause chronic bronchiolitis, which is an important topic for future investigations. A possible role for inflammation can also explain the reversibility of the airflow limitation observed in some LAM patients [15]. The recent discovery of an important role for the vitamin D-protein axis in LAM [16] can also be understood in the context of the known role of vitamin $\mathrm{D}$ on airway remodelling [17].

The observed association between the number of terminal bronchioles and the surface density renders further support to this hypothesis where more normal appearing lung parenchyma (i.e. higher surface density) shows a more preserved lung parenchyma compared to more diseased areas. However, even the more preserved lung parenchyma show an almost three-fold reduction in their surface density. This association between severity of cystic deformation of the parenchyma and the number of small airways is 

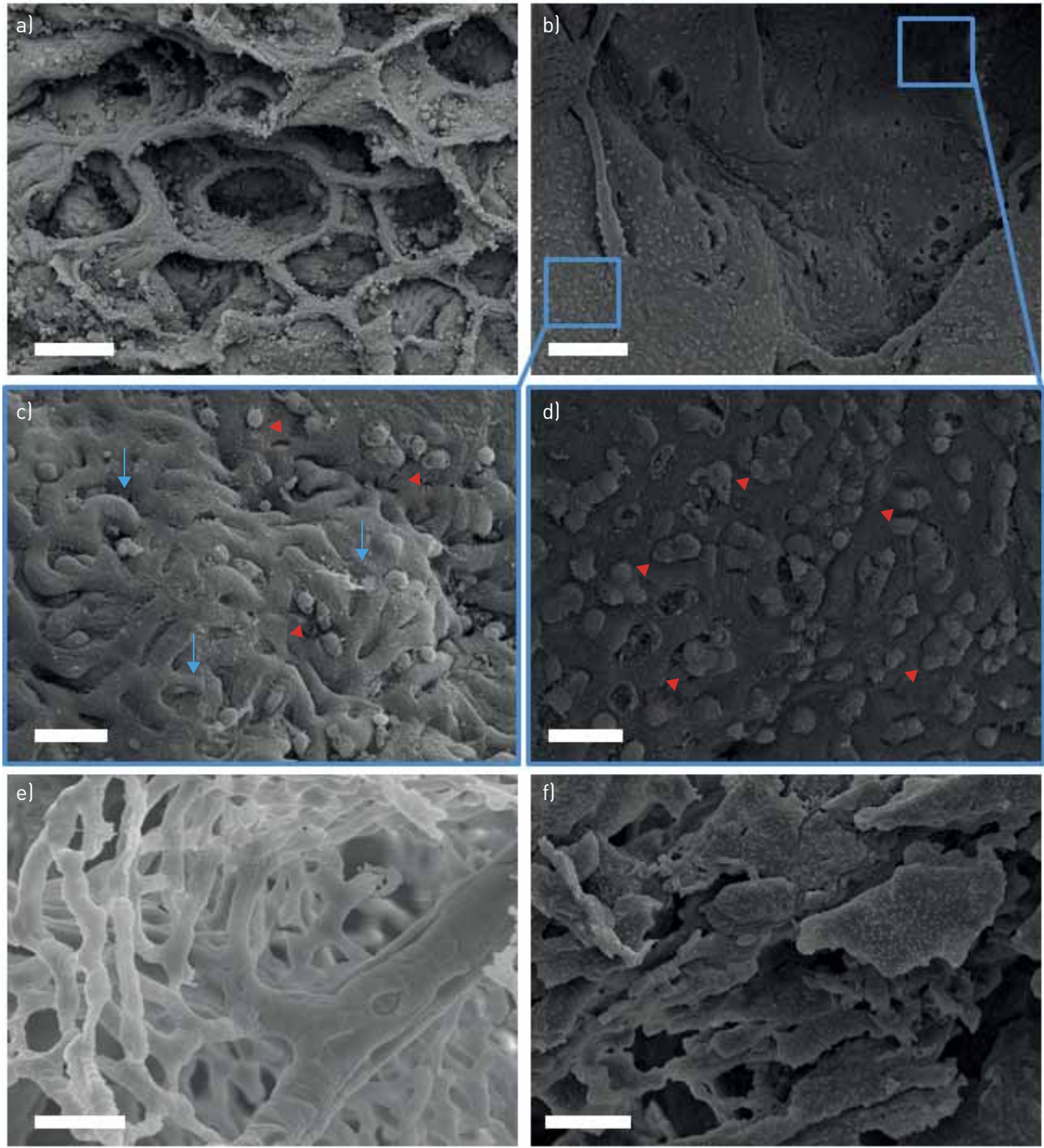

FIGURE 7 Structural morphology and vascularity of lymphangioleiomyomatosis (LAM). In comparison with the intact alveolar architecture of healthy control lungs (a), scanning electron micrographs of pulmonary LAM revealed large cystic honeycombing with $(c, d)$ cystic walls showed intermingled plumb, spindle-shaped cell proliferation (blue arrows) whereas the inner cystic wall is largely covered by type II pneumocytes (red arrowheads). e) Microvascular corrosion casting depicts the thin-walled alveolar plexus of healthy control tissue. f) Microvascular architecture in pulmonary LAM demonstrated a distinct aberrant vascularity with sinusoid-like vessel and missing hierarchy. Scale bars: (a and b) $100 \mu \mathrm{m}$; (c-f) $20 \mu \mathrm{m}$.

in line with longitudinal patient data from the NHLBI LAM registry where, in a large cohort of LAM patients the forced expiratory volume in $1 \mathrm{~s}$ decline was more rapid in patients with a greater profusion of parenchyma cysts [18], while the diffusion capacity also correlates with the disease extent seen on CT scans [19]. Nowadays, the cyst CT score and image texture are even used to predict disease progression and response to sirolimus [20]. Moreover, similar results were obtained using histological scoring where an association was found with semi-quantitative estimation of the degree of cystic lesions and infiltration of LAM cells and patient survival [21].

Limitations of this study include the relative low n-value. However, LAM is a rare disease and most descriptive studies are performed on similar numbers. Moreover, our CT and microCT measurements showed a very low inter-subject variability evident by the low standard deviations in all our analyses for the LAM group. Secondly, only end-stage lungs were investigated. Therefore, early manifestations of airway obstruction cannot be assessed. Thirdly, the control lungs were flushed prior to transplant, while the LAM lungs were not flushed; this probably explains the increased lung density of the LAM lungs. Lastly, this is 
also not a mechanistic study, as we primarily aimed to assess the degree of bronchiolar airway obstruction, the exact mechanisms and inhibitory possibilities are beyond the scope of this study.

In conclusion, LAM lungs show a three- to four-fold decrease in the number of airways from the seventh generation of airway branching. Airways of all sizes are lost by collapse of airways on the cysts and occlusion of the airways. The degree of parenchymal abnormality was strongly correlated with the number of airways. This is the first quantitative analysis of the degree of airway abnormality in LAM, showing the similarity in the degree of airway disease in end-stage COPD, thereby explaining the relentless pulmonary function decline. Therapeutic agents inhibiting airway loss are desperately needed to improve outcome in this rare disease.

Conflict of interest: None declared.

Support statement: S.E. Verleden is a post-doctoral fellow of the FWO (12G8718N) and acknowledges KU Leuven for their financial support (C24/18/073). W.A. Wuyts is supported by the Chrystal Chair of Boehringer Ingelheim. The special research fund of the Ghent University (BOF-UGent) is acknowledged for the financial support of the UGCT Center of Expertise (BOF.EXP.2017.0007). D.D. Jonigk is supported by the grant of the European Research Council (ERC); European Consolidator Grant, XHale (ref. no.771883). Funding information for this article has been deposited with the Crossref Funder Registry.

\section{References}

McCormack FX. Lymphangioleiomyomatosis: a clinical update. Chest 2008; 133: 507-516.

2 Henske EP, McCormack FX. Lymphangioleiomyomatosis - a wolf in sheep's clothing. J Clin Invest 2012; 122 : 3807-3816.

3 Baldi BG, Araujo MS, Freitas CSG, et al. Evaluation of the extent of pulmonary cysts and their association with functional variables and serum markers in lymphangioleiomyomatosis (LAM). Lung 2014; 192: 967-974.

4 Burger CD, Hyatt RE, Staats BA. Pulmonary mechanics in lymphangioleiomyomatosis. Am Rev Respir Dis 1991; 143: $1030-1033$

5 Sobonya RE, Quan SF, Fleishman JS. Pulmonary lymphangioleiomyomatosis: quantitative analysis of lesions producing airflow limitation. Hum Pathol 1985; 16: 1122-1128.

6 Hayashi T, Kumasaka T, Mitani K, et al. Bronchial involvement in advanced stage lymphangioleiomyomatosis: histopathologic and molecular analyses. Hum Pathol 2016; 50: 34-42.

7 Taveira-DaSilva AM, Hedin C, Stylianou MP, et al. Reversible airflow obstruction, proliferation of abnormal smooth muscle cells, and impairment of gas exchange as predictors of outcome in lymphangioleiomyomatosis. Am J Respir Crit Care Med 2001; 164: 1072-1076.

8 Verleden SE, Vasilescu DM, Willems S, et al. The site and nature of airway obstruction after lung transplantation. Am J Respir Crit Care Med 2014; 189: 292-300.

9 Boon M, Verleden SE, Bosch B, et al. Morphometric analysis of explant lungs in cystic fibrosis. Am J Respir Crit Care Med 2016; 193: 516-526.

10 McDonough JE, Yuan R, Suzuki M, et al. Small-airway obstruction and emphysema in chronic obstructive pulmonary disease. N Engl J Med 2011; 365: 1567-1575.

11 Verleden SE, Vasilescu DM, McDonough JE, et al. Linking clinical phenotypes of chronic lung allograft dysfunction to changes in lung structure. Eur Respir J 2015; 46: 1430-1439.

12 Masschaele B, Dierick M, Loo DV, et al. HECTOR: A 240kV micro-CT setup optimized for research. J Phys: Conf Ser 2013; 463: 12012.

13 Yushkevich PA, Piven J, Hazlett HC, et al. User-guided 3D active contour segmentation of anatomical structures: significantly improved efficiency and reliability. Neuroimage 2006; 31: 1116-1128.

14 Rodriguez A, Ehlenberger DB, Dickstein DL, et al. Automated three-dimensional detection and shape classification of dendritic spines from fluorescence microscopy images. PLoS One 2008; 3: e1997.

15 Taveira-DaSilva AM, Steagall WK, Rabel A, et al. Reversible airflow obstruction in lymphangioleiomyomatosis. Chest 2009; 136: 1596-1603.

16 Miller S, Coveney C, Johnson J, et al. The vitamin D binding protein axis modifies disease severity in lymphangioleiomyomatosis. Eur Respir J 2018; 52: 1800951.

17 Pfeffer PE, Hawrylowicz CM. Vitamin D in asthma: mechanisms of action and considerations for clinical trials. Chest 2018; 153: 1229-1239.

18 Gupta N, Lee H-S, Ryu JH, et al. The NHLBI LAM Registry: prognostic physiologic and radiologic biomarkers emerge from a 15-year prospective longitudinal analysis. Chest 2019; 155: 288-296.

19 Müller NL, Chiles C, Kullnig P. Pulmonary lymphangiomyomatosis: correlation of CT with radiographic and functional findings. Radiology 1990; 175: 335-339.

20 Gopalakrishnan V, Yao J, Steagall WK, et al. Use of CT imaging to quantify progression and response to treatment in lymphangioleiomyomatosis. Chest 2019; 155: 962-971.

21 Matsui K, Beasley MB, Nelson WK, et al. Prognostic significance of pulmonary lymphangioleiomyomatosis histologic score. Am J Surg Pathol 2001; 25: 479-484. 